\title{
SMART HOMES FOR A BETTER LIVING USING BLUETOOTH COMMUNICATION BASED ON ATMEGA MICROCONTROLLER
}

\author{
Monika Rana ${ }^{1}$, Ramandeep Singh ${ }^{2}$ \\ ${ }^{I}$ PG Student M.Tech (ES), EECE Department, ITM University, Gurgaon, Haryana, India \\ ${ }^{2}$ Assistant Professor, EECE Department, ITM University, Gurgaon, Haryana, India
}

\begin{abstract}
A smart home covers a variety of theoretical and practical approaches that deals with methodology of living today and in the future [1]. Technology has influenced and changed the life of humans in many ways. To design a device that will be serviceable to others is a huge contribution to the society [2]. Today mobile phones (smart phones, android etc.) can preforms almost all the tasks that once only PCs used to handle. With these advanced features and thought of elderly in mind, a device is designed. This device provides a much more advanced and a safer home to us. Smart home is a complex technology that uses information technology to control the electrical appliances and monitors the environment. The design and implementation presented in this paper is of a device which will use bluetooth technology for basic home automation and a wireless home network is desirable which does not incur any additional cost of wiring. The advantages and disadvantages are also discussed, along with the future scope and application areas.
\end{abstract}

Keywords: Bluetooth, Microcontroller, Home Appliance, Android, Smart homes.

\section{INTRODUCTION}

The idea of home automation was initially thought of to remotely turn devices on and off and thereafter with advancement the idea included provisions of complete access to devices while the user may be miles away from the home, may be elderly person or with a handicap. The implementation of home automation may utilize variety of technologies that may have come in market recently or have been in use over a longer period of time.

The home automation is made for several reasons of ease, security and energy efficiency. Home automation is used to control electric appliance like air conditioner, fan, bulb, gate and door lock etc. it can be control appliance within home or anywhere. Home automation of appliance may be wired or wireless. For our convenient we generally use wireless automation because of increasing smartphones and tablets in recent years. The smartphone provide easy connectivity with other devices. Through smartphones we can easily control appliance through Bluetooth, NFC, WLAN, mobile internet etc. if we want to control appliance from hundreds of mile away, we use internet to control our appliance and within home we generally use Bluetooth or NFC.

Bluetooth is a standard way to connect the devices and eliminate the wire between them. It is low power, low cost, and easily available device. Bluetooth is available in many device like smartphone, tablet etc. Bluetooth technology is easy to use and understand. Hence it could be used suitably in a cost effective manner.

The embedded system is connected to appliance in home and it is ready to receive control through bluetooth receiver. Any bluetooth enabled device (generally we use smartphone) is connected to that embedded system and send command for controlling home appliance. Bluetooth enabled Smartphone which is connected to embedded system and send control through application installed in phone and which is easily available on internet.

\section{BLOCK DIAGRAM}

The entire embedded system consists of two parts, one is android application and the other one is an electronic controlling circuitry. In the first part the android device is used for communicating with the controlling circuit through bluetooth channel. The android application required for this system can be easily downloaded and installed from Google Play or Play Store free of cost.

On the other hand the electronic circuitry consists of a microcontroller, a bluetooth module, relay driver IC along with relays which will switch electrical loads on the circuit and to switch the power supply. The android device sends a command which is received by the receiver of the bluetooth module and forwards it through its USART serial interface to the microcontroller. After receiving the command, the microcontroller performs the necessary action.

The microcontroller used in system is ATMEL's ATmega128, a high performance; low power RISC architecture based 8-bit MCU. The operating voltage of this microcontroller is $5 \mathrm{~V}$. So voltage regulator (LM78055) is used to get the desired voltage.

Whereas the bluetooth module (HC-05) run on $3.3 \mathrm{~V}$. This technology is a replacement for cables and wire harness [3]. It is an easy to use bluetooth serial port protocol module, designed for wireless serial connection setup. The UART of 
both the devices are in TTL logic, so we can directly connect receiver of Microcontroller to the transmitter of the Bluetooth Module through a current limiting resistor.

$\mathrm{R} 1, \mathrm{R} 2$ and R3 are the relays used to switch electrical load and power supply. Here driver relay IC (L293D) is used because the microcontroller operates on $5 \mathrm{~V}$ and cannot source sufficient current to drive the relays. All relays are rated $12 \mathrm{~V} \mathrm{DC}$. The COM and NO pins of the relays are used to connect the loads to be controlled.

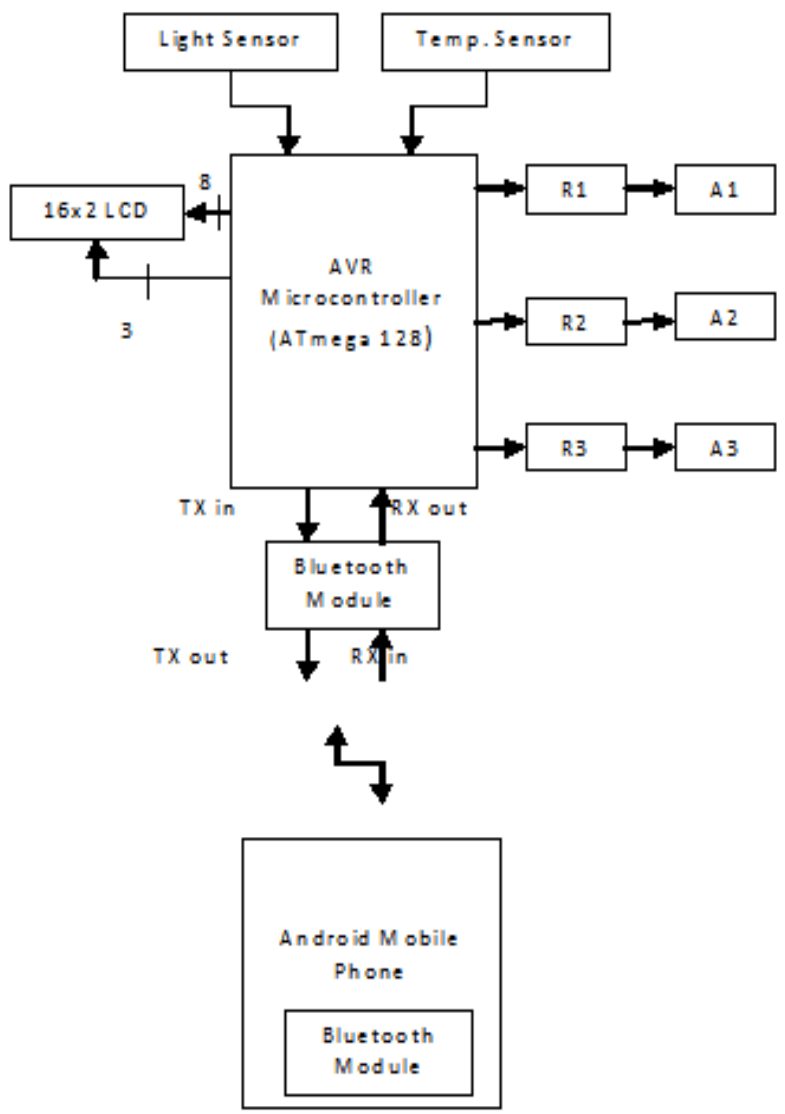

Fig 1.Block diagram of controller device

A1, A2 and A3 are the appliances which can be controlled by the android phone using bluetooth. The status of the appliances will be displayed on a $16 \times 2$ LCD screen. The LCD gets data update from the microcontroller and displays it.

A light sensor is also used for detecting light. It is a small sensor which changes its resistance when light falls on it. It is used to determine the intensity of light in a room, hall etc. A temperature sensor is used for determining the temperature. It gathers data (temperature) from the source and converts it into digital data which can be easily understood by the observer or the device.

\section{FLOW CHART/ALGORITHM}

The algorithm or flow for the working of the designed embedded system is as follows:-

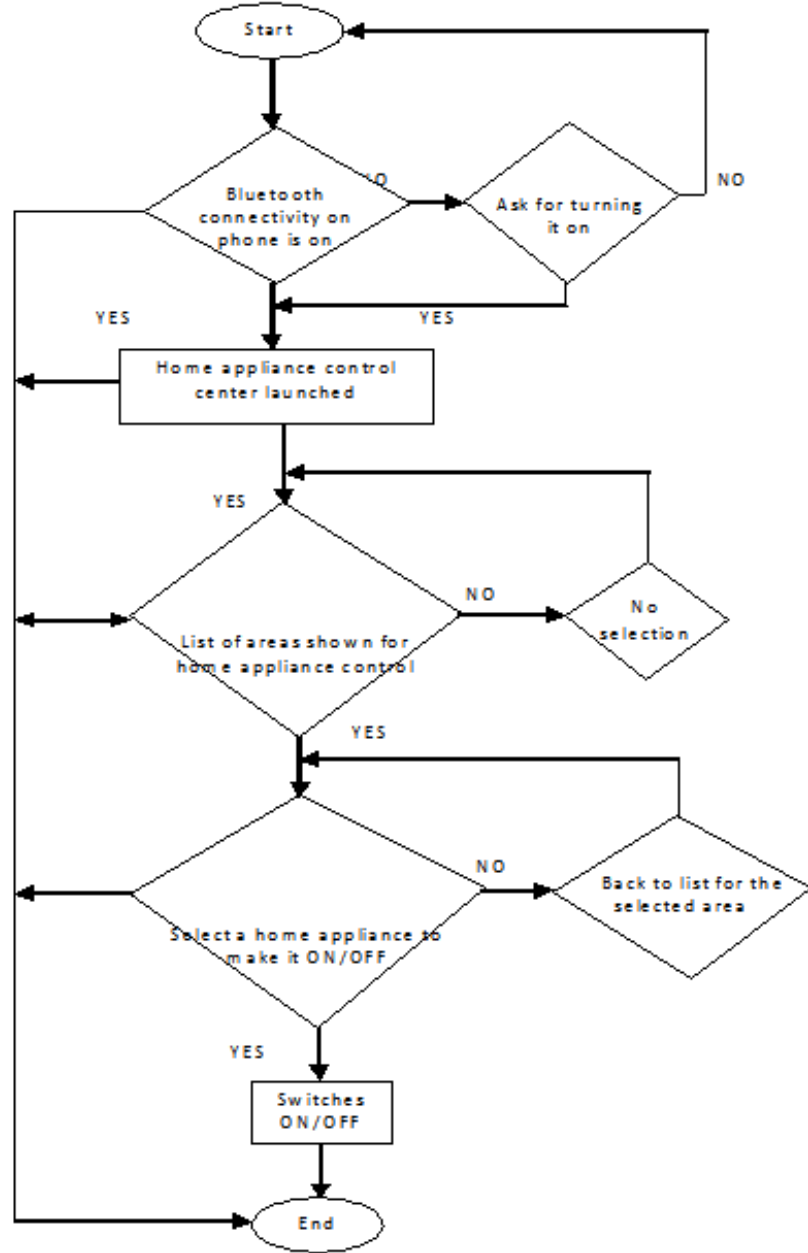

Fig 2 Flow Chart of the complete system

First of all we have to turn on the power of the device. When we turn on the power, the bluetooth connectivity of the phone is verified. If the bluetooth is not turned on the request for the same is issued. After the Bluetooth has been turned on or is already turned on, the home appliance control center is requested to display the list of areas under its control. If no areas are displayed under home appliance control center, the same is scanned over and over again. Otherwise a home appliance selected from the list of home appliance control center which the user may turn ON/OFF depending on its ideal state. The user may exit or return back to the list of areas for home appliance control.

\section{APPLICATION AREAS}

There are numerous applications of smart bluetooth technology, some of these are described below:-

a. Industrial Automation: - Industrial equipment's can now send key performance and safety information without the need of long and complex wiring. They can be directly connected to bluetooth smart ready phone, tablet or computers. It allows for higher flexibility and cut the cost down for machinery installation and the whole network can be easily integrated.

b. Healthcare and Fitness: - Home health monitoring [4] will be considered to keep the elderly healthy and 
active. Much medical equipment can be easily connected to smart phones wirelessly through bluetooth. Few such examples are the iHealth glucomonitoring system, electronic stethoscope model 3200 and the onyx II wireless pulse oximeter.

c. Proximity and Anti-Theft Accessories: - One easy way to keep an eye on our pets and kids is to tag them with a small bluetooth accessory which will generate an alert every time they sneak out of reach. Wallets, keys and luggage tagged with bluetooth can alert the smartphone if someone tries to steal them. There are applications such as FIND ME profile and Bluetooth also supports proximity which can seamlessly enable the above described applications.

\section{APPLIANCES}

Appliances are those intelligent artifacts that make the user's life more fascinating, interesting and comfortable. There are many different types of smart appliances in the market few of them include smart pen, gate reminder, smart pillow, smart refrigerator, smart greenhouse and many more [5]. These appliances make the life of people smarter and technology based.

\section{ADVANTAGES \& LIMITATIONS}

The main advantage of Bluetooth is that it is easily available and in reach of common people. It consumes very less battery power and requires less processing power for processing. The synced bluetooth devices does not need clear line of sight between them and the chances of other wireless networks interfering with bluetooth are very low. The disadvantage of bluetooth is that the battery usage during a single transfer is negligible, but if Bluetooth is kept on whole day, then battery loss is significant and because of this it inevitably eats into the battery of these devices, and lowers the battery life considerably. The other disadvantage of bluetooth is that the security in Bluetooth is good but it is much better in infrared technology.

\section{RESULTS}

The following are the result of the designed embedded system: -

When the system is switched on, a welcome message will be displayed on the LCD.

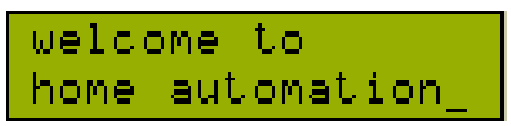

Fig 3 LCD screenshot 1

The next message to be displayed is the initial state of the devices.

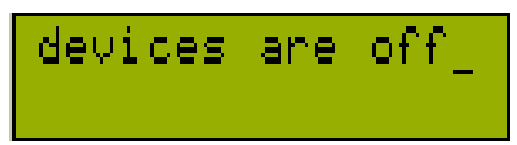

Fig 4 LCD screenshot 2
If the user has switched on the bulb, a message will be displayed on the LCD for the same.

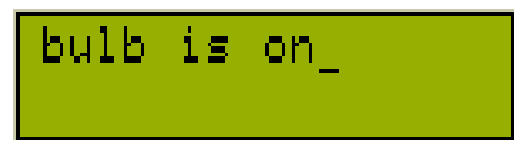

Fig 5 LCD screenshot 3

Now, if all the devices are on, the LCD will display a message notifying the status of the devices.

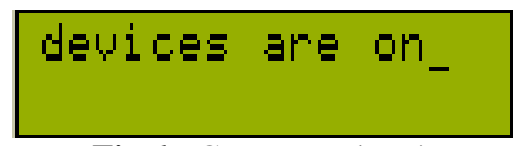

Fig 6 LCD screenshot 4

\section{CONCLUSIONS AND FUTURE SCOPE}

The goal of this paper was to outline the design and implementation of an embedded system which can easily interface with the existing home appliances and communicate with a smart phone via bluetooth using serial interfacing. We intended to create a home which more or less acts as a rational agent [6]. This research shows many types of applications for implementing home automation. The applications are not limited to those discussed in this paper. The technology discussed could be used in wide variety of applications that requires sensors and appliances and can serve the society in a very instructive manner. Our goal should not be limited to smart home technology but in future we should move past smart homes that automate every task for us [7].

\section{ACKNOWLEDGEMENTS}

The research paper is made possible through the help and support from my project head, lab faculty and the staff of Department of Electrical, Electronics \& Communication Engineering of ITM University, Gurgaon. I thank related projects and the large number of open sources that helped me throughout the research.

\section{REFERENCES}

[1] Dengler, Sebastian; Awad, Abdalkarim; Dressler, Falko, "Sensor/Actuator Networks in Smart Homes for Supporting Elderly and Handicapped People." Advanced Information Networking and Applications Workshops, 2007, AINAW '07. 21st International Conference on, Volume 2, 21-23 May 2007 Page(s):863 - 868 .

[2] Piyare, R and Tazil, M, "Bluetooth Based Home Automation System Using Cell Phone.” IEEE 15th International Symposium on Consumer Electronics (2011).

[3] Shepherd, R, "BIuetooth Wireless Technology in the Home." Electronics \& Communication Engineering Journal 13 (2001): 195-203. IEEE/IEE Electronic Library. 15 Oct. 2007. 
[4] T. Tamura, T. Togawa, M. Ogawa, and M. Yoda, "Fully automated health monitoring system in the home," Med. Eng. Physics, 20, pp. 573-579, 1998.

[5] Jiang, Li, Da-You Liu, and Bo Yang, "Smart Home Research." Machine Learning and Cybernetics (2004). 15 Oct. 2007.

[6] S. K. Das, D. J. Cook, A. Bhattacharya, E. O. Heierman, III, and T.-Y. Lin, "The Role of Prediction Algorithms on the MavHome Smart Home Architectures," IEEE Wireless Communications (Special Issue on Smart Homes), Vol. 9, No. 6, pp. 77-84, Dec. 2002.

[7] Yamazaki, T, "Beyond the Smart Home." Hybrid Information Technology, 2006. ICHIT'06. Vol 2. International Conference on, Volume 2, Nov. 2006 Page(s):350 - 355 .

\section{BIOGRAPHIES}

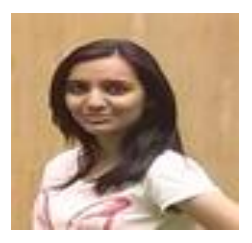

Monika Rana is currently Post Graduate student in Masters of Technology, Embedded Systems from ITM University, Gurgaon. She completed her B.Tech in Electronics and Communication Engineering from Banasthali University, Jaipur in 2012. Her area of interest is Embedded System Design and Real Time Systems.

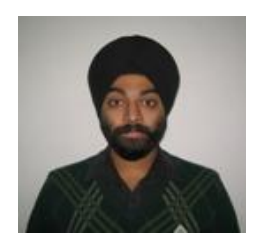

Ramandeep Singh is an Assistant Professor in EECE Department of ITM University, Gurgaon. $\mathrm{He}$ is pursuing Ph.D in embedded systems from ITM University. In 2009 he has completed M.E. in embedded systems. He is a B. Tech. graduate from GGSIPU, Delhi. His core research areas are low power embedded systems, robotics, FPGA based embedded systems and SCADA. Prior to joining ITM University he has worked with NXP Semiconductors, Bangalore. He has various publications in international journals and conferences. 